\title{
A THEOREM AND A COUNTEREXAMPLE IN THE THEORY OF SEMIGROUPS OF NONLINEAR TRANSFORMATIONS $\left(^{1}\right)$
}

\author{
BY \\ MICHAEL G. CRANDALL AND THOMAS M. LIGGETT
}

\begin{abstract}
This paper studies the basic method in current use for constructively obtaining a generator from a given semigroup of nonlinear transformations on a Banach space. The method is shown to succeed in real two-dimensional Banach spaces and to fail in a particular three-dimensional example. Other results of independent interest are obtained. For example, it is shown that the concepts of "maximal accretive" and "hyperaccretive" (equivalently, $m$-accretive or hypermaximal accretive) coincide in $R^{n}$ with the maximum norm.
\end{abstract}

Introduction. The fundamental open problem in the theory of semigroups of nonlinear transformations on a Banach space $X$ is that of determining whether or not a "generator" can be associated with each semigroup in such a way that the semigroup can be recovered from its generator. It is known that all the usual $X$-valued generators of such a semigroup $S$ can have empty domains, even if $S$ is strongly continuous and $S(t)$ is a contraction for each $t \geqq 0$ (see [3, $\S 4]$ ). However, if $X$ is a Hilbert space or if $X$ is finite dimensional, there is an accretive subset $A$ of $X \times X$ such that $S$ is given by

$$
S(t) x=\lim _{n \rightarrow \infty}\left(I+\frac{t}{n} A\right)^{-n} x, \quad t>0, x \in X,
$$

and, indeed, such a formula holds even for many semigroups $S$ which do not have generators in any usual sense. If $X$ is a Hilbert space, then $A$ is uniquely determined by $S$, but, unfortunately, this is not the case if $X$ is a general finite-dimensional Banach space (see [3, §4]). It would be extremely pleasant if, given $S$, one could constructively determine an $A$ for which (1) held. This would settle the representation question and at the same time would clarify the ambiguity concerning which (if any) of the possible $A$ 's satisfying (1) was the "correct" one in cases of nonuniqueness. The main results of this paper show that the approach to this problem which succeeds if $X$ is a Hilbert space (and in other special cases) also

Received by the editors September 15, 1970.

AMS 1969 subject classifications. Primary 4750; Secondary 4780, 3495.

Key words and phrases. Nonlinear semigroups, generator, accretive, extension theorem.

( $\left.{ }^{1}\right)$ Preparation of this paper was supported in part by the Office of Naval Research under Contract N00014-69-A-0200-4022 and the Air Force under Contract AF-AFOSR-69-1781. Reproduction in whole or in part is permitted for any purpose of the United States Government. Copyright (C) 1971, American Mathematical Society 
succeeds if $X$ is a (real) two-dimensional Banach space, but fails in general. Our proof of the two-dimensional result turns out to be more difficult than the proof of the same theorem in general Hilbert spaces (itself nontrivial), an indication of the fact that in contrast to the linear case, the theory of semigroups of nonlinear transformations is rich even in finite dimensions.

Precise definitions and descriptions of our main results are given below. If $C$ is a subset of $X$, a semigroup on $C$ is a function $S$ on $[0, \infty)$ such that $S(t)$ maps $C$ into $C$ for $t \geqq 0$ and satisfies

$$
S(t+\tau)=S(t) S(\tau) \text { for } t, \tau \geqq 0,
$$

and

$$
\lim _{t \downarrow 0} S(t) x=S(0) x=x \quad \text { for } x \in C .
$$

If $S$ is a semigroup on $C$ such that $\|S(t) x-S(t) y\| \leqq\|x-y\|$ for $t \geqq 0$ and $x, y \in C$ we will write $S \in Q(C)$. If $A \subseteq X \times X$, we define

$$
\begin{aligned}
A x & =\{y:[x, y] \in A\}, \\
D(A) & =\{x: A x \neq \varnothing\}, \\
R(A) & =\bigcup\{A x: x \in D(A)\} .
\end{aligned}
$$

If $B \subseteq X \times X$ and $\lambda$ is real, one sets

$$
\begin{aligned}
A+B & =\{[x, y+z]: y \in A x \text { and } z \in B x\}, \\
\lambda A & =\{[x, \lambda y]: y \in A x\}, \\
A^{-1} & =\{[y, x]:[x, y] \in A\} .
\end{aligned}
$$

A subset $B$ of $X \times X$ is called accretive if $(I+\lambda B)^{-1}$ is single-valued for $\lambda>0$ and

$$
\left\|(I+\lambda B)^{-1} x-(I+\lambda B)^{-1} y\right\| \leqq\|x-y\| \text { for } \lambda>0
$$

and $x, y \in D\left((I+\lambda B)^{-1}\right)$.

In [3], the present authors proved that if $A \subseteq X \times X$ is accretive and $R(I+\lambda A)$ $\supseteq \mathrm{Cl}(D(A))$ for all sufficiently small positive $\lambda$, then $\lim _{n \rightarrow \infty}(I+(t / n) \dot{A})^{-n} x$ exists for $t>0$ and $x \in \mathrm{Cl}(D(A))$. Moreover, if $S(t) x$ is defined as the above limit, then $S \in Q(\mathrm{Cl}(D(A)))$. The only approach which has been used to date to show that a given $S \in Q(C)$ can be written in the form (1) for some accretive $A$ is described in detail in $\S 3$ of [3]. Briefly, one assumes $C$ is closed and convex and defines maps $J_{\lambda, t}: C \rightarrow C$ for $\lambda, t>0$ by

$$
J_{\lambda, t} x=\left(I+\lambda\left(\frac{I-S(t)}{t}\right)\right)^{-1} x
$$

$J_{\lambda, t}$ is well defined and a contraction, its definition being equivalent to

$$
J_{\lambda, t} x=\frac{t}{\lambda+t} x+\frac{\lambda}{\lambda+t} S(t) J_{\lambda, t} x
$$


for $x \in C$. According to Theorem III of [3], if

$$
\lim _{t \downarrow 0} J_{\lambda, t} x
$$

exists for $\lambda>0$ and $A$ is defined by

$$
(I+\lambda A)^{-1} x=\lim _{t \downarrow 0} J_{\lambda, t} x,
$$

then (1) holds. If $X$ is a Hilbert space this limit always exists (see [7] and [9]). In general, it has been shown only that $J_{\lambda, t} x$ is bounded as $t \downarrow 0$ and that $\lim _{(\lambda, t) \rightarrow(0,0)}\left\|J_{\lambda, t} x-x\right\|=0$ (see [3]). This was used in [3] to show, among other things, that if $X$ is finite dimensional and $S \in Q(C)$, then (1) holds for some $A$, since in that case it sufficed to obtain a sequence $\left\{t_{n}\right\}, t_{n} \downarrow 0$, for which $J_{\lambda, t_{n}}$ converged. This procedure is, of course, not constructive and has little chance of extending to general $X$. Also, we remark that many works have employed the maps $J_{\lambda, t}$ under assumptions of various kinds (see [4], [11], [12], [15] for example) related to the question of whether the limit (3) exists. Thus it is of considerable interest to determine whether or not (3) always exists. The answer is quite surprising. If $\operatorname{dim} X=1$, then $X$ is a Hilbert space, so the limit (3) exists by previous results. In $\S 1$, we will show that if the (real) dimension of $X$ is 2 , then the limit (3) exists. The arguments are quite different from those used in the Hilbert space case. Finally, in $\$ 2$, we give an example of an $S \in Q(X)$, where $X=R^{3}$ with the norm $\|(a, b, c)\|$ $=\max (|a|,|b|,|c|)$, such that the limit (3) does not exist. The reason for this difference is essentially that the surface of the unit ball of a Banach space can be much more complicated in three dimensions than in two. The role of the smoothness of unit balls is also illustrated by the fact that the limit (3) always exists if $X$ is finite dimensional and $X^{*}$ is strictly convex.

Much of $\S 2$ is devoted to proving a theorem of independent interest, which is used to extend a function defined on a subset of $[0, \infty) \times X$ to a semigroup $S \in Q(X)$. This theorem states that if $X=R^{d}$ with the maximum norm and $A$ is maximal with respect to inclusion among accretive sets, then $R(I+\lambda A)=X$ for $\lambda>0$. The same result is shown to fail if $X=R^{2}$ with the $p$-norm, $1<p<\infty, p \neq 2$.

1. The two-dimensional theorem. We will need a variety of preliminary results. The first lemma implies that $S(t) x$ is Lipschitz continuous in $t$ uniformly for $x$ in compact subsets of the relative interior of $C$, provided that $S \in Q(C)$ and $X$ is finite dimensional. We will call $S \in Q(C)$ uniformly continuous if $\lim _{t \downarrow}\|S(t) x-x\|$ $=0$ holds uniformly for $x$ in bounded subsets of $C$. Clearly every $S \in Q(C)$ is uniformly continuous if $X$ is finite dimensional.

Lemma 1.1. Let $S \in Q(C)$ be uniformly continuous, and let $Y$ denote the smallest closed flat space (translate of a subspace of $X$ ) containing $C$. Then for each $\varepsilon>0$ there is a number $M \geqq 0$ such that

$$
\|S(t+\tau) x-S(t) x\| \leqq \tau M
$$


for $t, \tau \geqq 0$ and all $x \in C$ which satisfy

$$
\|x\|<\varepsilon^{-1} \text { and }\|x-y\|>\varepsilon \text { for } y \in Y \backslash C .
$$

Proof. For $x \in C, \tau>0, t \geqq 0$ and $k$ a positive integer we have

$$
\begin{aligned}
\|S(t+\tau) x-S(t) x\| & \leqq\|S(\tau) x-x\| \leqq \tau\|S(\tau) x-x\| / \tau \\
& \leqq \tau\left(\frac{1}{k} \sum_{i=0}^{k-1} \frac{\|S((i+1) \tau / k) x-S(i \tau / k) x\|}{(\tau / k)}\right) \leqq \tau \frac{\|S(\tau / k) x-x\| .}{(\tau / k)} .
\end{aligned}
$$

Hence to prove the lemma, it suffices to show that $\lim \sup _{t \downarrow 0}(\|S(t) x-x\| / t)$ is uniformly bounded for $x$ satisfying the assumptions of the lemma. Next, let $u, v, w \in X$ and $1 \geqq \kappa \geqq 0$. Then

$$
\|u\|=\|u+\kappa(v-u)-\kappa(v-u)\| \leqq \kappa\|v-u\|+\|(1-\kappa) u+\kappa v\|
$$

and

$$
\|v\|=\|v+(1-\kappa)(u-v)-(1-\kappa)(u-v)\| \leqq(1-\kappa)\|u-v\|+\|(1-\kappa) u+\kappa v\|
$$

imply, after adding and rearranging, that

$$
\|u\|+\|v\|-\|u-v\| \leqq 2\|(1-\kappa) u+\kappa v\| .
$$

Given $x \in C$ satisfying $\|x\|<\varepsilon^{-1}$ and $\|x-z\|>\varepsilon$ for $z \in Y \backslash C$, assume $\|S(t) x-x\|>0$ and define

$$
y=x-\varepsilon(S(t) x-x) /\|S(t) x-x\| .
$$

Note that $y \in C$, since $y \in Y$ and $\|x-y\|=\varepsilon$. Let $k$ be a positive integer and set

$$
u=x-S(t) x, \quad v=x-S(k t) y, \quad \kappa=\|x-S(t) x\| /(\varepsilon+\|x-S(t) x\|)
$$

in (1.4) to obtain

$$
\begin{aligned}
&\|x-S(k t) y\|+\|x-S(t) x\|-\|S(t) x-S(k t) y\| \\
& \leqq 2(\|x-S(t) x\| /(\varepsilon+\|x-S(t) x\|))\|y-S(k t) y\| .
\end{aligned}
$$

Using $S \in Q(C),(1.6)$ implies

$$
\begin{aligned}
\|x-S(k t) y\|-\|x-S((k-1) t) y\| & +\|x-S(t) x\| \\
& \leqq 2 \varepsilon^{-1}(\|x-S(t) x\|)\|y-S(k t) y\| .
\end{aligned}
$$

Next sum the inequalities (1.7) for $1 \leqq k \leqq m$ to obtain

$$
\begin{aligned}
\|x-S(m t) y\|-\|x-y\|+m \| x & -S(t) x \| \\
& \leqq 2 \varepsilon^{-1}\|x-S(t) x\|\left(\sum_{k=1}^{m}\|y-S(k t) y\|\right) .
\end{aligned}
$$

Noticing that $\|y\| \leqq \varepsilon+\varepsilon^{-1}$ by (1.5), define

$$
g(\tau)=\sup \left\{\|z-S(\alpha) z\|: z \in C,\|z\| \leqq \varepsilon+\varepsilon^{-1}, 0 \leqq \alpha \leqq \tau\right\} .
$$


The uniform continuity of $S$ implies $g$ is continuous at $\tau=0$. Since

$$
\|x-S(m t) y\|-\|x-y\| \geqq-\|y-S(m t) y\| \geqq-g(m t),
$$

(1.8) implies

$$
(\|x-S(t) x\| / t)(1-2 g(m t) / \varepsilon) \leqq g(m t) / m t .
$$

Choose $\delta>0$ such that $g(\delta)<\varepsilon / 4$. If $0<t<\delta / 2$, there is an integer $m$ such that $\delta / 2<m t<\delta$ and so, by (1.9), $\|x-S(t) x\| / t \leqq \varepsilon / \delta$ if $0<t<\delta / 2$. Since $g$ depends only on $\varepsilon$, the proof is complete.

REMARK. The same proof shows $\|x-S(t) x\| / t$ is bounded if $x$ is in the relative interior of $C$ and $S$ is uniformly continuous on some relative neighborhood of $x$.

REMARK. Y. Kômura informed us he had previously established (unpublished) a version of Lemma 1.1.

To continue, we define the (multi-valued) duality map of $X$ into $X^{*}$ by

$$
F(x)=\left\{f \in X^{*}: f(x)=\|x\|^{2}=\|f\|^{2}\right\} .
$$

It is obvious that $F(x)$ is nonempty, closed, convex and bounded for each $x \in X$. If $S \in Q(C)$ we define its strong generator $B$ by

$$
B x=\lim _{t \downarrow 0} \frac{S(t) x-x}{t}
$$

provided the limit exists. The following simple and well-known lemma implies $-B$ is accretive (or equivalently, that $B$ is dissipative).

Lemma 1.10. Suppose $S \in Q(C)$ and $x, y \in D(B)$. Then $f(B x-B y) \leqq 0$ for each $f \in F(x-y)$.

Proof. For $t>0$,

$$
f(S(t) x-S(t) y) \leqq\|f\|\|S(t) x-S(t) y\| \leqq\|x-y\|^{2}=f(x-y)
$$

so

$$
f((S(t) x-x) / t-(S(t) y-y) / t) \leqq 0,
$$

and the lemma follows upon letting $t \downarrow 0$.

We will require two further elementary facts for the proof of the main result of this section.

LEMMA 1.11. Let $X$ be a real two-dimensional Banach space and $x \in X$ be such that $F(x)$ has exactly two distinct extreme points $g$ and $h$. If $x_{n} \rightarrow x, h\left(x_{n}\right)<g\left(x_{n}\right)$ for each $n$, and $f_{n} \in F\left(x_{n}\right)$, then $f_{n} \rightarrow g$.

Proof. We first remark that if $X$ is two dimensional then $F(x)$ is either a line segment or a point, so the lemma concerns the only nontrivial case. Since $g$ and $h$ 
are linearly independent and $X^{*}$ is two dimensional, we may write $f_{n}=a_{n} g+b_{n} h$ for some $a_{n}, b_{n} \in R$. From

$$
\left(a_{n}+b_{n}\right)\|x\|^{2}=a_{n} g(x)+b_{n} h(x)=f_{n}(x) \leqq\|x\|\left\|x_{n}\right\|
$$

and

$$
a_{n} g\left(x_{n}\right)+b_{n} h\left(x_{n}\right)=f_{n}\left(x_{n}\right)=\left\|x_{n}\right\|^{2}
$$

it follows that

$$
\left(a_{n}+b_{n}\right)\|x\| \leqq\left\|x_{n}\right\| \text { and } a_{n}+b_{n} \rightarrow 1 .
$$

Moreover, we see any limit point of $\left\{f_{n}\right\}$ is in $F(x)$ and hence is a convex combination of $g$ and $h$. Thus it suffices to show $\left\{b_{n}\right\}$ has no positive limit points. Writing

$$
\left\|x_{n}\right\|^{2}=\left(a_{n}+b_{n}\right) g\left(x_{n}\right)+b_{n}\left(h\left(x_{n}\right)-g\left(x_{n}\right)\right),
$$

using $g\left(x_{n}\right) \leqq\left\|x_{n}\right\|\|x\|$ and (1.12), we see that $b_{n}\left(h\left(x_{n}\right)-g\left(x_{n}\right)\right) \geqq 0$, so $b_{n} \leqq 0$ for all sufficiently large $n$, and we are done.

Finally we state, without proof, a simple lemma from real analysis.

LEMMA 1.13. Let $r$ be a real-valued absolutely continuous function on $[0,1]$. For a real number $c$ set $\Omega=\{\tau \in[0,1]: r(\tau)>c\}$. Then

$$
\int_{\Omega} r^{\prime}(\tau) d \tau=\max (r(1), c)-\max (r(0), c) .
$$

Moreover, $r^{\prime}(\tau)=0$ for almost all $\tau \in\{s \in[0,1]: r(s)=c\}$.

We are now ready to prove the main result of this section.

THEOREM 1.14. Let $X$ be a real two-dimensional Banach space and $C$ a closed convex subset of $X$. If $S \in Q(C), \lambda>0, z \in C$ and $J_{\lambda, t} z \in C$ is defined by

$$
J_{\lambda, t} z=\frac{t}{t+\lambda} z+\frac{\lambda}{t+\lambda} S(t) J_{\lambda, t} z
$$

then $\lim _{t \downarrow 0} J_{\lambda, t} z$ exists.

Proof. For simplicity we will take $C=X$ in the proof. This allows us to avoid an uninteresting technical point in the argument. Since $J_{\lambda, t} z$ remains bounded as $t \downarrow 0$, it suffices to show it has a unique limit point. The proof is by contradiction, so we assume there are sequences $s_{m} \downarrow 0$ and $t_{n} \downarrow 0$ such that $J_{\lambda, s_{m}} z \rightarrow u, J_{\lambda, t_{n}} z \rightarrow v$ and $u \neq v$. We will set

$$
u_{m}=J_{\lambda, s_{m}} z \text { and } \quad v_{n}=J_{\lambda, t_{n}} z \text {. }
$$

Since $X$ is two dimensional, $F(v-u)$ has at most two extreme points, which will be denoted by $g$ and $h$. (If $F(v-u)$ is a singleton, we take $g=h$.) We choose $g$ and $h$ so that

$$
g(v-z) \geqq h(v-z)
$$


Define $f \in X^{*}$ by

$$
f=g-h \text {. }
$$

Setting $c=g(v)-h(v)=g(u)-h(u)$ we note that (1.16), (1.17) imply

$$
f(z) \leqq c .
$$

We adopt the following notation:

$$
\begin{aligned}
R^{-} & =\{x \in X: f(x)<c\}, \quad R^{+}=\{x \in X: f(x)>c\}, \\
R^{0} & =\{x \in X: f(x)=c\} .
\end{aligned}
$$

For $i \in\{-, 0,+\}$, we define

$$
\begin{aligned}
A_{m}^{i} & =\left\{\tau \in[0,1]: S\left(s_{m} \tau\right) u_{m} \in R^{i}\right\}, \\
B_{n}^{i} & =\left\{\tau \in[0,1]: S\left(t_{n} \tau\right) v_{n} \in R^{i}\right\} .
\end{aligned}
$$

Passing to subsequences if necessary, we may assume

$$
\mu\left(A_{m}^{i}\right) \rightarrow \alpha^{i}, \quad \mu\left(B_{n}^{i}\right) \rightarrow \beta^{i},
$$

where $\mu$ is Lebesgue measure. Clearly $\alpha^{i} \geqq 0, \beta^{i} \geqq 0$ for $i \in\{-, 0,+\}$ and

$$
\alpha^{-}+\alpha^{0}+\alpha^{+}=\beta^{-}+\beta^{0}+\beta^{+}=1 \text {. }
$$

Let $B$ be the strong generator of $S$. For $i \in\{-, 0,+\}$ we define

$$
\begin{aligned}
G_{v}^{i} & =\lim _{\varepsilon \downarrow 0} \sup \left\{g(B y) \mid\|y-v\|<\varepsilon, y \in R^{i} \cap D(B)\right\}, \\
G_{u}^{i} & =\lim _{\varepsilon \downarrow 0} \inf \left\{g(B x) \mid\|x-u\|<\varepsilon, x \in R^{i} \cap D(B)\right\}, \\
H_{v}^{i}= & \lim _{\varepsilon \downarrow 0} \sup \left\{h(B y) \mid\|y-v\|<\varepsilon, y \in R^{i} \cap D(B)\right\}, \\
H_{u}^{i}= & \lim _{\varepsilon \downarrow 0} \inf \left\{h(B x) \mid\|x-u\|<\varepsilon, x \in R^{i} \cap D(B)\right\} .
\end{aligned}
$$

In the above definitions we use the conventions inf $\varnothing=\infty$, sup $\varnothing=-\infty$. In this connection, we also agree that $0( \pm \infty)=0$. The reader may note that if $\pm \infty$ occurs in an inequality below, the inequality is trivial, and that infinite quantities are multiplied by zero in the calculations and cause no subsequent problems. (It is probably best to assume that all quantities are finite while reading the proof. The other cases are essentially simpler.)

The first main step is to derive a collection of inequalities relating the above quantities. We claim that

$$
\begin{array}{rlr}
H_{v}^{-} \leqq H_{u}^{-} & H_{v}^{-} \leqq H_{u}^{0} & H_{v}^{-} \leqq H_{u}^{+} \\
G_{v}^{-} \leqq G_{u}^{-} & H_{v}^{0} \leqq H_{u}^{0} & H_{v}^{0} \leqq H_{u}^{+} \\
G_{v}^{0} \leqq G_{u}^{-} & G_{v}^{0} \leqq G_{u}^{0} & H_{v}^{+} \leqq H_{u}^{+} \\
G_{v}^{+} \leqq G_{u}^{-} & G_{v}^{+} \leqq G_{u}^{0} & G_{v}^{+} \leqq G_{u}^{+}
\end{array}
$$


The proofs of these inequalities are all quite similar, and we do only some of them here. Consider, for example, $G_{v}^{+} \leqq \min \left\{G_{u}^{-}, G_{u}^{0}, G_{u}^{+}\right\}$. Any infinite quantity is on the correct side of the inequality, so it suffices to show $x_{m} \rightarrow u, y_{n} \rightarrow v, x_{m}, y_{n} \in D(B)$ and $f\left(y_{n}\right)>c$ for each $n$ implies

$$
\limsup _{n \rightarrow \infty} g\left(B y_{n}\right) \leqq \liminf _{m \rightarrow \infty} g\left(B x_{m}\right) .
$$

For such sequences, choose $g_{n, m} \in F\left(y_{n}-x_{m}\right)$. If $g_{n} \in F\left(y_{n}-u\right), g_{n} \rightarrow g$ by Lemma 1.13. Moreover, every limit point of $\left\{g_{n, m}\right\}_{m=1}^{\infty}$ is in $F\left(y_{n}-u\right)$ (because $y_{n}-x_{m}$ $\rightarrow y_{n}-u$ as $m \rightarrow \infty$ ), so we have that

$$
\lim _{n \rightarrow \infty} \limsup _{m \rightarrow \infty}\left\|g_{n, m}-g\right\|=0 .
$$

By Lemma $1.10, g_{n, m}\left(B y_{n}\right) \leqq g_{n, m}\left(B x_{m}\right)$ for each $m, n$. This, together with (1.22) and the fact that $\left\{B y_{n}\right\}$ and $\left\{B x_{m}\right\}$ are bounded (by Lemma 1.1) implies that (1.21) holds. As another example, we verify $H_{v}^{0} \leqq H_{u}^{0}$ assuming both these numbers are finite and $g \neq h$. Here, if $y_{n}, x_{m} \in R^{0}$, then

$$
f\left(y_{n}\right)=f\left(x_{m}\right) \text { and } f=g-h
$$

implies $y_{n}-x_{m}$ is in the (one-dimensional) null space of $g-h$, so $y_{n}-x_{m}$ is a scalar multiple of $v-u$. Since $y_{n} \rightarrow v, x_{m} \rightarrow u, y_{n}-x_{m}=a_{n, m}(v-u)$ where $a_{n, m} \rightarrow 1$ as $n, m \rightarrow \infty$. Hence $F\left(y_{n}-x_{m}\right)=a_{n, m} F(v-u)$, and by Lemma 1.10 we have $a_{n, m} h\left(B y_{n}\right)$ $\leqq a_{n, m} h\left(B x_{m}\right)$, so $H_{v}^{0} \leqq H_{u}^{0}$ follows at once.

We need another type of inequality. According to Lemma 1.1, the functions $S(\tau) x$ are uniformly Lipschitz continuous in $\tau$ for $x$ in bounded subsets of $X$, and hence are differentiable almost everywhere in $\tau$. From (1.15) we have

so

$$
\lambda^{-1}\left(v_{n}-z\right)=\left(S\left(t_{n}\right) v_{n}-v_{n}\right) / t_{n}
$$

$$
\begin{aligned}
\lambda^{-1} g\left(v_{n}-z\right) & =\frac{1}{t_{n}} \int_{0}^{t_{n}} \frac{d}{d \tau} g\left(S(\tau) v_{n}\right) d \tau \\
& =\int_{0}^{1} g\left(B S\left(t_{n} \tau\right) v_{n}\right) d \tau=\sum_{i} \int_{B_{n}^{l}} g\left(B S\left(t_{n} \tau\right) v_{n}\right) d \tau,
\end{aligned}
$$

where $\sum_{i}$ denotes a sum over $i \in\{-, 0,+\}$. According to Lemma 1.13,

$$
\int_{B_{n}^{0}} f\left(B S\left(t_{n} \tau\right) v_{n}\right) d \tau=0
$$

or

$$
\int_{B_{n}^{0}} g\left(B S\left(t_{n} \tau\right) v_{n}\right) d \tau=\int_{B_{n}^{0}} h\left(B S\left(t_{n} \tau\right) v_{n}\right) d \tau .
$$

Since $v_{n} \rightarrow v$ and $\mu\left(B_{n}^{0}\right) \rightarrow \beta^{0}$, this implies

$$
\underset{n \rightarrow \infty}{\limsup } \int_{B_{n}^{0}} g\left(B S\left(t_{n} \tau\right) v_{n}\right) d \tau \leqq \beta^{0} \min \left(G_{v}^{0}, H_{v}^{0}\right) .
$$


Taking the lim sup as $n \rightarrow \infty$ in (1.23) and using (1.24) yields

$$
\lambda^{-1} g(v-z) \leqq \beta^{-} G_{v}^{-}+\beta^{0} \min \left(G_{v}^{0}, H_{v}^{0}\right)+\beta^{+} G_{v}^{+} .
$$

In order to compare (1.25) and an expression involving $u$, we need to work a little harder. By Lemma 1.13, we have

$$
\int_{A_{m}^{+}} f\left(B S\left(s_{m} \tau\right) u_{m}\right) d \tau=\left(s_{m}\right)^{-1}\left(\max \left[f\left(S\left(s_{m}\right) u_{m}\right), c\right]-\max \left[f\left(u_{m}\right), c\right]\right) .
$$

The right-hand side of (1.26) is nonnegative if $f\left(S\left(s_{m}\right) u_{m}-u_{m}\right) \geqq 0$, or, by (1.15), if $f\left(u_{m}-z\right) \geqq 0$. Since $u_{m} \rightarrow u, f(u)=c$, and $f(z) \leqq c$ by (1.18), this is true for large $m$ unless $f(z)=c$ and $f\left(u_{m}\right)<c$. But then (1.15) implies $f\left(S\left(s_{m}\right) u_{m}\right) \leqq f\left(u_{m}\right)<c$ and the right-hand side of $(1.26)$ is 0 . Thus

$$
\underset{m \rightarrow \infty}{\liminf } \int_{A_{m}^{+}} f\left(B S\left(s_{m} \tau\right) u_{m}\right) d \tau \geqq 0 .
$$

The same argument shows

$$
\underset{n \rightarrow \infty}{\liminf } \int_{B_{n}^{+}} f\left(B S\left(t_{n} \tau\right) v_{n}\right) d \tau \geqq 0 .
$$

Using (1.20) and (1.27), we have

$$
\liminf _{m \rightarrow \infty} \int_{A_{m}^{+}} g\left(B S\left(s_{m} \tau\right) u_{m}\right) d \tau \geqq \liminf _{m \rightarrow \infty} \int_{A_{m}^{+}} h\left(B S\left(s_{m} \tau\right) u_{m}\right) d \tau \geqq \alpha^{+} H_{u}^{+} \geqq \alpha^{+} H_{v}^{0} .
$$

Hence

$$
\begin{aligned}
\lambda^{-1} g(u-z) & =\lim _{m \rightarrow \infty} \lambda^{-1} g\left(u_{m}-z\right) \\
& =\liminf _{m \rightarrow \infty} \sum_{i} \int_{A_{m}^{i}} g\left(B S\left(s_{m} \tau\right) u_{m}\right) d \tau \\
& \geqq \alpha^{-} G_{u}^{-}+\alpha^{0} G_{u}^{0}+\alpha^{+} \max \left(G_{u}^{+}, H_{v}^{0}\right) .
\end{aligned}
$$

Since $g(v-u)=\|v-u\|>0,(1.25)$ and (1.29) imply that

$$
\alpha^{-} G_{u}^{-}+\alpha^{0} G_{u}^{0}+\alpha^{+} \max \left(G_{u}^{+}, H_{v}^{0}\right)<\beta^{-} G_{v}^{-}+\beta^{0} \min \left(G_{v}^{0}, H_{v}^{0}\right)+\beta^{+} G_{v}^{+} .
$$

A similar argument shows that

$$
\alpha^{-} H_{u}^{-}+\alpha^{0} \max \left(H_{u}^{0}, G_{u}^{0}\right)+\alpha^{+} H_{u}^{+}<\beta^{-} H_{v}^{-}+\beta^{0} H_{v}^{0}+\beta^{+} \min \left(H_{v}^{+}, G_{u}^{0}\right) .
$$

It remains to show the relations (1.19), (1.20), (1.30) and (1.31) are inconsistent. Since $\max \left(G_{v}^{-}, G_{v}^{0}, G_{v}^{+}\right) \leqq G_{u}^{-},(1.30)$ and (1.19) imply

$$
\alpha^{0} G_{u}^{0}+\alpha^{+} \max \left(G_{u}^{+}, H_{v}^{0}\right)<\left(\alpha^{0}+\alpha^{+}\right) G_{u}^{-}
$$

and hence $\alpha^{0}+\alpha^{+}>0$. Similarly, $\beta^{0}+\beta^{+}>0$, and we can define

$$
\bar{G}=\left(\beta^{0} \min \left(H_{v}^{0}, G_{v}^{0}\right)+\beta^{+} G_{v}^{+}\right) /\left(\beta^{0}+\beta^{+}\right) .
$$


Then, using $G_{v}^{0} \leqq G_{u}^{0}, G_{v}^{+} \leqq G_{u}^{0}$, we see $\bar{G} \leqq G_{u}^{0}$ and using $G_{v}^{+} \leqq G_{u}^{+}$yields $\bar{G} \leqq \max \left(G_{u}^{+}, H_{v}^{0}\right)$. This implies

$$
\left(\alpha^{0}+\alpha^{+}\right) \bar{G} \leqq \alpha^{0} G_{u}^{0}+\alpha^{+} \max \left(G_{u}^{+}, H_{v}^{0}\right) .
$$

This last inequality, (1.30) and the definition of $\bar{G}$ imply

$$
\alpha^{-} G_{u}^{-}+\left(\alpha^{0}+\alpha^{+}\right) \bar{G}<\beta^{-} G_{v}^{-}+\left(\beta^{0}+\beta^{+}\right) \bar{G} .
$$

Since $G_{v}^{-} \leqq G_{u}^{-}$and $\bar{G} \leqq G_{u}^{-}$this implies $\alpha^{-}<\beta^{-}$. A similar argument using (1.31) shows that $\beta^{-}<\alpha^{-}$, and this is the desired contradiction. The proof is complete.

2. The three-dimensional counterexample. The counterexample to be presented in this section will be given explicitly only on part of its domain. Taking $X=R^{3}$ with norm

$$
\left\|\left(x^{(1)}, x^{(2)}, x^{(3)}\right)\right\|=\max \left\{\left|x^{(1)}\right|,\left|x^{(2)}\right|,\left|x^{(3)}\right|\right\}
$$

we will give a sequence $\left\{t_{n}\right\}$ of positive numbers which converge to zero and a sequence $\left\{f_{n}\right\}$ of functions, $f_{n}: I_{n} \rightarrow X$ where $I_{n}=\left[0, t_{n}\right]$, with the following properties:

(2.1) $\left\|f_{n}(t+\tau)-f_{m}(t+\eta)\right\| \leqq\left\|f_{n}(\tau)-f_{m}(\eta)\right\|$ if $t, \tau, \eta \geqq 0$ and $t+\tau \in I_{n}, t+\eta \in I_{m}$,

(2.2) $f_{n}(0)+\left(f_{n}(0)-f_{n}\left(t_{n}\right)\right) / t_{n}=(-6,18,-30)$, and

(2.3) $\left\{f_{n}(0)\right\}_{n=1}^{\infty}$ has $(0,0,0)$ and $(1,1,1)$ as limit points.

Condition (2.1) guarantees that setting

$$
S(t) f_{n}(\tau)=f_{n}(t+\tau), \quad t, \tau \geqq 0, t+\tau \in I_{n}, n=1,2, \ldots,
$$

is consistent with the requirement that $S \in Q(X)$. Condition (2.2) implies that if $S$ satisfies (2.4), then

$$
J_{1, t}(-6,18,-30)=\left(I+\left(I-S\left(t_{n}\right)\right) / t_{n}\right)^{-1}(-6,18,-30)=f_{n}(0) .
$$

Finally, (2.3) implies that $\lim _{t \downarrow 0} J_{1, t}(-6,18,-30)$ does not exist. In this partially defined form, the counterexample shows, at least, that one would have to use the long time behavior of $S(t)$ or the assumption that $S(t)$ is defined on $X$ (or a convex $C)$ to prove that $\lim _{t \downarrow 0} J_{1, t} x$ exists. To obtain the stronger result that no such proof is possible, we will first prove two extension theorems which will imply that there is an $S \in Q(X)$ satisfying (2.4) for our $\left\{f_{n}\right\}$. The example itself may be read independently of the extension theorems, and is given explicitly at the end of this section.

Below, $X=R^{d}$ with the norm

$$
\left\|\left(a^{(1)}, \ldots, a^{(d)}\right)\right\|=\max \left\{\left|a^{(i)}\right|: 1 \leqq i \leqq d\right\} .
$$

A set $A \subseteq X \times X$ is said to be maximal accretive if $A$ is accretive and $A$ is not a proper subset of another accretive set.

Theorem 2.5. Let $A \subseteq X \times X$. Then $A$ is maximal accretive if and only if $A$ is accretive and $R(I+\lambda A)=X$ for all $\lambda>0$. 
Proof. One direction is trivial. If $A$ is accretive and $R(I+\lambda A)=X$ for some $\lambda>0,(I+\lambda A)^{-1}$ is an everywhere-defined contraction. If $B$ is another accretive set and $B \supseteq A$, then $(I+\lambda B)^{-1}$ is a contraction which extends the everywheredefined $(I+\lambda A)^{-1}$. Hence $(I+\lambda A)^{-1}=(I+\lambda B)^{-1}$, so $A=B$.

The proof of the converse assertion will be based on an application of the Eilenberg-Montgomery fixed point theorem for multi-valued functions [5]. Since $A$ is maximal accretive if and only if $\lambda A$ is maximal accretive, it suffices to prove the theorem for $\lambda=1$.

Fix $u \in X$. We will show that $u \in R(I+A)$. For each $[x, y] \in A$, define

$$
V([x, y])=\{z \in X \mid\{[x, y],[z, u-z]\} \text { is accretive }\} .
$$

Suppose that

$$
\bigcap\{V([x, y]):[x, y] \in A\} \neq \varnothing .
$$

Then for $z$ in this intersection, $\{[z, u-z]\} \cup A$ will be accretive and therefore $[z, u-z] \in A$ by the maximality of $A$. But this says $u=z+(u-z) \in R(I+A)$. To show that (2.6) is true, we first note that $V([x, y])$ is closed, and if $z \in V([x, y])$ then $T=\{[x, y],[z, u-z]\}$ is accretive so

$$
\|z-x\|=\left\|(I+T)^{-1}(z+(u-z))-(I+T)^{-1}(x+y)\right\| \leqq\|u-(x+y)\| .
$$

Therefore $V([x, y])$ is compact in $X$, and (2.6) is equivalent to the statement that $\{V([x, y]):[x, y] \in A\}$ has the finite intersection property. We will use the following equivalent definition of accretive:

$A$ is accretive if and only if $\left\langle y_{1}-y_{2}, x_{1}-x_{2}\right\rangle_{s} \geqq 0$ whenever $\left[x_{i}, y_{i}\right] \in A, i=1,2$, where $\langle,\rangle_{s}: X \times X \rightarrow R$ is defined by

$$
\langle u, v\rangle_{s}=\sup \{f(u): f \in F(v)\} .
$$

The equivalence is proved in [8] for general Banach spaces $X$. In our case the equivalence is apparent.

Let $\left\{\left[x_{j}, y_{j}\right] \mid 1 \leqq j \leqq n\right\}$ be a finite subset of $A$. Given $b, c \in X$ we define the "cube"

$$
M(b, c)=\left\{a \in X \mid b^{(i)} \leqq a^{(i)} \leqq c^{(i)} \text { for } 1 \leqq i \leqq d\right\} .
$$

Let $M=M(b, c)$ be any such cube containing all the points $u-y_{k}, k=1, \ldots, n$, and define sets $T_{k}(x)$ for $0 \leqq k \leqq n$ and $x \in X$ by

$$
T_{0}(x)=M, \quad T_{k}(x)=\left\{z \in M \mid \min _{1 \leqq j \leqq k}\left\langle(u-z)-y_{j}, x-x_{j}\right\rangle_{s} \geqq 0\right\} .
$$

Clearly $T_{0}(x) \supseteq T_{1}(x) \supseteq \cdots \supseteq T_{n}(x)$. The condition $x \in T_{n}(x)$ is equivalent to $x \in \bigcap_{j=1}^{n} V\left(\left[x_{j}, y_{j}\right]\right)$, so we are done if we can show the map $x \rightarrow T_{n}(x)$ has a fixed point. Clearly $T_{n}$ defines a mapping of $M$ into subsets of $M$ which is continuous in the sense that $\left\{[x, y] \in M \times M: y \in T_{n}(x)\right\}$ is closed in $M \times M$. If we show that $T_{n}(x)$ is nonempty and contractible for each $x \in M$, the conditions of the main 
theorem of [5] will be satisfied and the multi-valued map $T_{n}$ will have a fixed point, thus completing the proof. Fix $x \in X$ and define $v_{k}=v_{k}(x)=\left(v_{k}^{(1)}, \ldots, v_{k}^{(d)}\right)$ for $1 \leqq k \leqq n$ by

$$
\begin{aligned}
v_{k} & =0 & & \text { if } x=x_{k} \\
v_{k}^{(i)} & =\left(x^{(i)}-x_{k}^{(i)}\right) /\left\|x-x_{k}\right\|^{2} & & \text { if } 0<\left|x^{(i)}-x_{k}^{(i)}\right|=\left\|x-x_{k}\right\|, \\
& =0 & & \text { if }\left|x^{(i)}-x_{k}^{(i)}\right|<\left\|x-x_{k}\right\| .
\end{aligned}
$$

Next we define maps $\rho_{k}$ on $T_{k-1}(x)$ for $1 \leqq k \leqq n$ by

$$
\rho_{k}(z)=z+v_{k} \min \left\{0,\left\langle(u-z)-y_{k}, x-x_{k}\right\rangle_{s}\right\} \text {. }
$$

Clearly $\rho_{k}$ is continuous (since $\langle,\rangle_{s}: X \times X \rightarrow R$ is continuous in its first argument), and $\rho_{k}$ is the identity on $T_{k}(x)$. We will show that $\rho_{k}$ maps into $T_{k}(x)$, and this will complete the proof, for then

$$
\rho_{n} \circ \rho_{n-1} \circ \cdots \circ \rho_{1}: M \rightarrow T_{n}(x)
$$

is the identity on $T_{n}(x)$. In particular, $T_{n}(x)$ is nonempty and is a retraction of $M$, so it is contractible (and hence acyclic).

Before continuing, let us observe that if $X^{*}$ is taken as $R^{d}$ with the norm $\left\|\left(a^{(1)}, \ldots, a^{(d)}\right)\right\|=\sum_{i=1}^{d}\left|a^{(i)}\right|$ then

$$
F\left(x-x_{k}\right)=\text { convex hull of }\left\{\left\|x-x_{k}\right\|^{2} v_{k}^{(j)} f_{j}: 1 \leqq j \leqq d\right\}
$$

where $f_{j}^{(i)}=\delta_{i j}$ for $1 \leqq i, j \leqq d$. Choosing $z \in T_{k-1}(x) \backslash T_{k}(x)$ we first verify that $\rho_{k}(z) \in M=M(b, c)$. Set $w=\rho_{k}(z)$. According to $(2.7)$, if $v_{k}^{(i)}=0$ then $w^{(i)}=z^{(i)}$ so $b^{(i)} \leqq w^{(i)} \leqq c^{(i)}$. If $v_{k}^{(i)} \neq 0$, we have $f=\left\|x-x_{k}\right\|^{2} v_{k}^{(i)} f_{i} \in F\left(x-x_{k}\right)$ and applying this to (2.7) gives

$$
\left(x^{(i)}-x_{k}^{(i)}\right) w^{(i)}=\left(x^{(i)}-x_{k}^{(i)}\right) z^{(i)}+\left\langle(u-z)-y_{k}, x-x_{k}\right\rangle_{s}<\left(x^{(i)}-x_{k}^{(i)}\right) z^{(i)}
$$

and, since $\left\langle(u-z)-y_{k}, x-x_{k}\right\rangle_{s} \geqq f\left((u-z)-y_{k}\right)$,

$$
\left(x^{(i)}-x_{k}^{(i)}\right) w^{(i)} \geqq\left(x^{(i)}-x_{k}^{(i)}\right)\left(u^{(i)}-y_{k}^{(i)}\right) .
$$

These inequalities imply $w^{(i)}$ is between $z^{(i)}$ and $\left(u^{(i)}-y_{k}^{(i)}\right)$. Since $z, u-y_{k} \in M(b, c)$, $b^{(i)} \leqq w^{(i)} \leqq c^{(i)}$ and $w \in M$. It remains to verify that $w \in T_{k}(x)$. Choose $f \in F\left(x-x_{k}\right)$ so that $f\left((u-z)-y_{k}\right)=\left\langle(u-z)-y_{k}, x-x_{k}\right\rangle_{s}$. Then

$$
\begin{aligned}
\left\langle(u-w)-y_{k}, x-x_{k}\right\rangle_{s} & \geqq f\left((u-w)-y_{k}\right) \\
& =f\left((u-z)-y_{k}\right)-f\left(v_{k}\right)\left\langle(u-z)-y_{k}, x-x_{k}\right\rangle_{s} \\
& =\left\langle(u-z)-y_{k}, x-x_{k}\right\rangle_{s}-\left\langle(u-z)-y_{k}, x-x_{k}\right\rangle_{s}=0
\end{aligned}
$$

since $f\left(v_{k}\right)=1$ for $f \in F\left(x-x_{k}\right)$.

Now fix $j<k$ and consider two cases:

Case (i). Suppose $\left\|x_{j}-x_{k}\right\|<\left\|x_{j}-x\right\|+\left\|x-x_{k}\right\|$. Since $z \in T_{k-1}(x)$ there is an $\in F\left(x-x_{j}\right)$ so that $f\left((u-z)-y_{j}\right) \geqq 0$. We may assume $f$ is an extreme point of 
$F\left(x-x_{j}\right)$. If $f\left(v_{k}\right)<0$, then $f\left(x-x_{k}\right)=-\left\|x-x_{k}\right\|\left\|x-x_{j}\right\|$ and, therefore,

$$
\begin{aligned}
f\left(x_{k}-x_{j}\right) & =f\left(x_{k}-x\right)+f\left(x-x_{j}\right) \\
& =\|x-x,\|\left(\left\|x-x_{k}\right\|+\left\|x-x_{j}\right\|\right)>\left\|x-x_{j}\right\|\left\|x_{k}-x_{j}\right\|,
\end{aligned}
$$

which is impossible since $\|f\|=\left\|x-x_{j}\right\|$. Hence, $f\left(v_{k}\right) \geqq 0$. But then

$$
f\left((u-w)-y_{j}\right)=f\left((u-z)-y_{i}\right)-f\left(v_{k}\right)\left\langle(u-z)-y_{k}, x-x_{k}\right\rangle_{s} \geqq f\left((u-z)-y_{i}\right) \geqq 0,
$$

so $\left\langle(u-w)-y_{j}, x-x_{j}\right\rangle_{s} \geqq 0$.

Case (ii). Suppose $\left\|x_{k}-x_{j}\right\|=\left\|x_{k}-x\right\|+\left\|x-x_{j}\right\|$. Since $A$ is accretive,

$$
\left\langle y_{k}-y_{i}, x_{k}-x_{j}\right\rangle_{s} \geqq 0,
$$

and there is an $f \in F\left(x_{k}-x_{j}\right)$ so that $f\left(y_{k}\right) \geqq f\left(y_{j}\right)$. Since the triangle equality holds above it is easy to see that

$$
\frac{\left\|x_{k}-x\right\|}{\left\|x_{k}-x_{j}\right\|} f \in F\left(x_{k}-x\right) \text { and } \frac{\left\|x-x_{j}\right\|}{\left\|x_{k}-x_{j}\right\|} f \in F\left(x-x_{j}\right)
$$

if $x_{k} \neq x_{j}\left(x_{k}=x_{j}\right.$ is a trivial subcase). Therefore

$$
\begin{aligned}
f(w) & =f(z)+f\left(v_{k}\right)\left\langle(u-z)-y_{k}, x-x_{k}\right\rangle_{s} \\
& =f(z)-\frac{\left\|x_{k}-x_{j}\right\|}{\left\|x_{k}-x\right\|}\left\langle(u-z)-y_{k}, x-x_{n}\right\rangle_{s} \\
& \leqq f(z)+\frac{\left\|x_{k}-x_{j}\right\|}{\left\|x_{k}-x\right\|} \frac{\left\|x_{k}-x\right\|}{\left\|x_{k}-x_{j}\right\|} f\left((u-z)-y_{k}\right) \\
& =f\left(u-y_{k}\right) \leqq f\left(u-y_{j}\right) .
\end{aligned}
$$

Hence $f\left((u-w)-y_{j}\right) \geqq 0$ which implies $\left\langle(u-w)-y_{j}, x-x_{j}\right\rangle_{s} \geqq 0$. The verification that $w=\rho_{k}(z) \in T_{k}(x)$ is complete, and the theorem is proved.

THEOREM 2.8. Suppose $A \subset X \times X$ is an accretive set which satisfies

$$
\sup \{\|y\|: y \in R(A)\}<\infty .
$$

Then there is a maximal accretive set $B$ with the following properties:

(i) $B \supseteq A$.

(ii) $D(B)=X$.

(iii) If $M(b, c)$ is the smallest cube containing $R(A)$, then $R(B) \subseteq M(b, c)$.

Proof. By Zorn's lemma, there is a set $B$ maximal with respect to inclusion among accretive sets satisfying (i) and (iii). Given $x \in X$ and $[w, z] \in B$, set

$$
T([w, z])=\left\{y \in M(b, c) \mid\langle y-z, x-w\rangle_{s} \geqq 0\right\} .
$$

Adopting the notation of the proof of Theorem 2.5 with $u=0$, if $\left[x_{i}, y_{i}\right] \in B$, $1 \leqq i \leqq n$, then

$$
\bigcap_{i=1}^{n} T\left(\left[x_{i}, y_{i}\right]\right)=-T_{n}(x) .
$$


It therefore follows from the proof of Theorem 2.5 that $\{T([w, z]):[w, z] \in B\}$ has the finite intersection property. Hence $\bigcap\{T([w, z]):[w, z] \in B\}$ is nonempty, and if $y$ is in this intersection, then $[x, y] \in B$ by maximality. Since $x$ was arbitrary, (ii) follows. In a similar way, the proof of Theorem 2.5 shows that if $u \in X$ then there is an $x \in X$ such that $B \cup[x, u-x]$ is accretive and $x \in u-M(b, c)=$ $\{u-y: y \in M(b, c)\}$. Hence $u-x \in M(b, c)$ and $[x, u-x] \in B$ by maximality. So $R(I+B)=X$ and $B$ is maximal accretive by the argument at the beginning of the proof of Theorem 2.5 .

REMARKS. Extension problems play an important role in parts of the theory of semigroups of linear and nonlinear transformations and their generators. In [13], Phillips proved that if $X$ is a Hilbert space and $A: X \rightarrow X$ is accretive, linear and densely defined then $R(I+A)=X$ if and only if $A$ is maximal among accretive linear operators. In [10], Minty showed that if $X$ is a Hilbert space and $A \subseteq X \times X$ is accretive (equivalently, monotone), then $A$ is maximal if and only if $R(I+A)=X$. In [9], Y. Kômura proved that if $S$ is a "maximal" semigroup of (nonlinear) contractions on a subset of a Hilbert space, then $S$ is defined on a convex set. However, we are aware of no results of this kind outside of Hilbert spaces (except in the theory of monotone sets, e.g. [2], which is not relevant to our work if $X$ is not a Hilbert space), nor do we know of any negative results. The problem of extending accretive sets is related (but probably not equivalent) to the problem of extending contractions, where there are strong negative results, see [6] and [14]. It seems likely that similar theorems are true in the accretive case. We give an example:

Let $X=R^{2}$ with the norm $\|(a, b)\|=\left(|a|^{p}+|b|^{p}\right)^{1 / p}$ for $1 \leqq p \leqq \infty$. Taking $p=1$ or $\infty$ we have $A \subseteq X \times X$ maximal accretive if and only if $R(I+A)=X$ by Theorem 2.5. The same is true for $p=2$ by Minty's theorem. For all $1 \leqq p \leqq \infty$ the set

$$
A=\{[(0,0),(0,0)],[(0,1),(1,0)],[(1,0),(0,-1)]\}
$$

is accretive. Let $\Delta_{1}, \Delta_{2}$ denote the open triangles

$$
\begin{aligned}
& \Delta_{1}=\{(a, b): a, b>0, a+b<1, b>a\}, \\
& \Delta_{2}=\{(a, b): a, b>0, a+b<1, a>b\} .
\end{aligned}
$$

It is straightforward to check that if $B \supseteq A$ and $B$ is accretive, then $D(B) \cap \Delta_{1}=\varnothing$ if $2<p<\infty$ and $D(B) \cap \Delta_{2}=\varnothing$ if $1<p<2$. It is known that if $B$ is accretive, $X$ is uniformly convex and $R(I+\lambda B) \supseteq$ convex closure of $D(B)$ for $\lambda>0$, then $\mathrm{Cl}(D(B))$ is convex. (See the final remarks of [1].) Clearly, then, there are no maximal accretive sets $B$ such that $B \supseteq A$ and $R(I+B)=X$ if $p \neq 1,2, \infty$.

We turn, at last, to the counterexample. Here we take $X=R^{3}$ with the maximum norm. For $\alpha, \beta \in X$ let $l(\alpha, \beta)$ be the line segment

$$
l(\alpha, \beta)=\{\alpha+\tau(\beta-\alpha): 0 \leqq \tau \leqq 1\}
$$


joining $\alpha$ and $\beta$. If $v$ is a positive multiple of $\beta-\alpha$, let $l(\alpha, \beta: v)$ denote the function

$$
g(t)=\alpha+t v, \quad 0 \leqq t \leqq\|\beta-\alpha\| /\|v\| .
$$

The sum $l(\alpha, \beta: v)+l(\beta, \gamma: w)+\cdots$ will denote the piecewise linear function of $t$ defined by starting from $\alpha$ at $t=0$, travelling toward $\beta$ with velocity $v$ until $\beta$ is reached, then travelling toward $\gamma$ with velocity $w$ until $\gamma$ is reached, etc. Let

$$
\begin{aligned}
& t_{n}=10^{-5 n} \quad \text { if } n \text { is odd, } \\
& =10^{-5 n-3} \text { if } n \text { is even; } \\
& \alpha_{n}=\frac{t_{n}}{1+t_{n}}(-6,18,0) \quad \text { if } n \text { is odd, } \\
& =(1,1,1)+\frac{t_{n}}{1+t_{n}}(5,0,-19) \text { if } n \text { is even; } \\
& \beta_{n}=\frac{t_{n}}{1+t_{n}}(0,0,10) \quad \text { if } n \text { is odd, } \\
& =(1,1,1)+\frac{t_{n}}{1+t_{n}}\left(4,-\frac{17}{3}, 4\right) \quad \text { if } n \text { is even; } \\
& \gamma_{n}=\frac{t_{n}}{1+t_{n}}\left(\frac{6}{7}, \frac{5}{7}, \frac{80}{7}\right)+\frac{t_{n}^{2}}{1+t_{n}}\left(\frac{9}{7}, \frac{15}{14}, \frac{15}{7}\right) \quad \text { if } n \text { is odd, } \\
& =(1,1,1)+\frac{t_{n}}{1+t_{n}}\left(\frac{23}{6}, \frac{-119}{18}, \frac{25}{6}\right)+\frac{t_{n}^{2}}{1+t_{n}}\left(-\frac{1}{4},-\frac{17}{12}, \frac{1}{4}\right) \text { if } n \text { is even; } \\
& \delta_{n}=\frac{t_{n}}{1+t_{n}}\left(-\frac{6}{7},-1, \frac{200}{7}\right)+\frac{t_{n}^{2}}{1+t_{n}}\left(\frac{-9}{7}, \frac{-3}{2}, \frac{195}{7}\right) \quad \text { if } n \text { is odd, } \\
& =(1,1,1)+\frac{t_{n}}{1+t_{n}}\left(\frac{73}{6}, \frac{-289}{18}, \frac{25}{2}\right)+\frac{t_{n}^{2}}{1+t_{n}}\left(\frac{49}{4}, \frac{-187}{12}, \frac{51}{4}\right) \quad \text { if } n \text { is even; } \\
& \varepsilon_{n}=t_{n}(0,0,30) \quad \text { if } n \text { is odd, } \\
& =(1,1,1)+t_{n}(12,-17,12) \text { if } n \text { is even; } \\
& v_{1 n}=(18,-54,30) \quad \text { if } n \text { is odd, } \\
& =(-3,-17,69) \text { if } n \text { is even; } \\
& v_{2 n}=(18,15,30) \quad \text { if } n \text { is odd, } \\
& =(-3,-17,3) \quad \text { if } n \text { is even; } \\
& v_{3 n}=(-3,-3,30) \quad \text { if } n \text { is odd, } \\
& =(15,-17,15) \quad \text { if } n \text { is even; } \\
& v_{4 n}=(18,21,30) \quad \text { if } n \text { is odd, } \\
& =(-3,-17,-9) \text { if } n \text { is even. }
\end{aligned}
$$

Next, define

$$
f_{n}=l\left(\alpha_{n}, \beta_{n}: v_{1 n}\right)+l\left(\beta_{n}, \gamma_{n}: v_{2 n}\right)+l\left(\gamma_{n}, \delta_{n}: v_{3 n}\right)+l\left(\delta_{n}, \varepsilon_{n}: v_{4 n}\right) .
$$

The domain of $f_{n}$ turns out to be $\left[0, t_{n}\right]$, and the sequence $\left\{f_{n}\right\}$ has the properties (2.1), (2.2) and (2.3), as may be seen by straightforward but tedious checking. An 
important fact to notice is that if $x \in l\left(\gamma_{2 n+1}, \delta_{2 n+1}\right)$ and $y \in l\left(\gamma_{2 m}, \delta_{2 m}\right)$ for some $n, m$, and $f \in F(y-x)$, then the first coordinate of $f$ is zero. This fact follows from the way $\left\{t_{n}\right\}$ was chosen. To obtain an $S \in Q(X)$ such that (2.4) holds, we note that

$$
A=\bigcup_{n=1}^{\infty}\left\{\left[f_{n}(t),-D^{+} f_{n}(t)\right]: 0 \leqq t<t_{n}\right\}
$$

is accretive, where $D^{+}$denotes the right derivative. Indeed, $A$ is accretive if and only if (2.1) holds. According to Theorems 2.5 and 2.8, there is an accretive set $B$, $B \supseteq A$, such that $D(B)=X$ and $R(I+\lambda B)=X$ for $\lambda>0$. So, by Theorem I of [3]

$$
S(t) x=\lim _{n \rightarrow \infty}\left(I+\frac{t}{n} B\right)^{-1} x
$$

exists and $S \in Q(X)$. Finally, Theorem II of [3] implies that (2.4) holds.

\section{REFERENCES}

1. H. Brezis and A. Pazy, Accretive sets and differential equations in Banach spaces, Israel J. Math. 8 (1970), 367-383.

2. F. E. Browder, Nonlinear maximal monotone operators in Banach space, Math. Ann. 175 (1968), 89-113. MR 36 \#6989.

3. M. Crandall and T. Liggett, Generation of semi-groups of nonlinear transformations on general Banach spaces, Amer. J. Math. (to appear).

4. J. Dorroh, Some classes of semi-groups of nonlinear transformations and their generators, J. Math. Soc. Japan 20 (1968), 437-455. MR 37 \#6796.

5. S. Eilenberg and D. Montgomery, Fixed point theorems for multi-valued transformations, Amer. J. Math. 68 (1946), 214-222. MR 8, 51.

6. B. Grünbaum, On a theorem of Kirszbraun, Bull. Res. Council Israel Sect. F7 (1957/58), 129-132. MR 21 \#5155.

7. T. Kato, Note on the differentiability of nonlinear semi-groups, Proc. Sympos. Pure Math., vol. 16, Amer. Math. Soc., Providence, R. I., 1964, pp. 91-94.

8. - Nonlinear semigroups and evolution equations, J. Math. Soc. Japan 19 (1967), 508-520. MR 37 \#1820.

9. Y. Kômura, Differentiability of nonlinear semigroups, J. Math. Soc. Japan 21 (1969), 375-402. MR 40 \#3358.

10. G. J. Minty, Monotone (nonlinear) operators in Hilbert space, Duke Math. J. 29 (1962), 341-346. MR 29 \#6319.

11. J. W. Neuberger, An exponential formula for one-parameter semi-groups of non-linear transformations, J. Math. Soc. Japan 18 (1966), 154-157. MR 34 \#622.

12. S. Oharu, Note on the representation of semi-groups of non-linear operators, Proc. Japan Acad. 42 (1966), 1149-1154. MR 36 \#3167.

13. R. S. Phillips, Dissipative operators and hyperbolic systems of partial differential equations, Trans. Amer. Math. Soc. 90 (1959), 193-254. MR 21 \#3669.

14. S. O. Schönbeck, On the extension of Lipschitz maps, Ark. Mat. 7 (1967), 201-209. MR 39 \#3297.

15. G. F. Webb, Representation of semi-groups of nonlinear nonexpansive transformations in Banach spaces, J. Math. Mech. 19 (1969/70), 159-170. MR 40 \#793.

\section{UNIVERSITY OF CALIFORNIA,}

Los Angeles, California 90024 